\title{
THE DIAGNOSIS OF CONGENITAL HEART DISEASE IN INFANTS BY CATHETERIZATION AND SELECTIVE ANGIOCARDIOGRAPHY
}

\author{
BY

\begin{abstract}
IB BOESEN, JOHN LIND, B. MERRILD-HANSEN, TH. ROSENDAL, OLE STORM, AND CARL WEGELIUS
\end{abstract} \\ From Dronning Louises Børnehospital, Copenhagen, Denmark, and the Wenner-Gren Cardiovascular Research \\ Laboratory at Norrtull's Hospital, Stockholm, Sweden
}

Received August 22, 1955

The anæsthetic and surgical techniques are now so highly developed that it is possible to operate on congenital heart disease even in infants. This is important progress, as about half the children with congenital malformations of the heart die before the age of one year: $30-40$ per cent of these suffer from conditions that can theoretically be corrected by operation with the possibilities that now exist-a percentage that will probably increase in the years to come. It is thus an urgent task to try to diagnose these malformations as early as possible as surgical correction is frequently necessary and may be accomplished safely and successfully in this age group.

A prerequisite for surgical treatment is an accurate diagnosis. The diagnostic methods are clinical, heart catheterization, and angiocardiography. The routine clinical diagnosis consists of case history, auscultation, electrocardiography, rœntgen examination, etc. Assisted by these simple means it is often possible to make a diagnosis correct enough for a decision whether the malformation can be treated by surgery or not, and whether this is desirable. In older children with congenital heart disease about two-thirds have such characteristic clinical findings that a comparatively satisfactory diagnosis can be made, for instance, with persistent patent ductus arteriosus, coarctation of the aorta, the tetralogy of Fallot. It is, however, necessary in a number of patients to use more complicated methods, heart catheterization and angiocardiography, which have proved of very great value for a correct anatomical and functional knowledge of the nature of congenital malformations of the heart and their influence on the circulation.

The younger the children with congenital heart diseases, the more difficult it is to make a relatively sure diagnosis on clinical findings alone. This is partly because there are far more types represented in the younger age group, viz., those serious conditions in which the children die early. The incidence of combined lesions is high and often accounts for the serious difficulties in early life. Besides, the general X-ray examination is not of the same value in infants as in later years, and the X-ray appearance of the heart and the great vessels in different heart lesions is often not characteristic at this age. Fluoroscopy is difficult because of the crying and lack of co-operation by the child, because of the physiological tachycardia, and because the thymus often overlaps the base of the heart and the great vessels.

The size of the heart is in our opinion of value in the diagnosis, and the rœntgenological determination of the heart volume is the most accurate method available. The cardiothoracic ratio, still widely in use, is unreliable (Lind, 1950). A heart that is large enough to suggest a doubtful prognosis is a main indication for investigation in acyanotic congenital lesions. The lack of differential criteria in the exact diagnosis of the cardiac malformation in infants with large hearts and evidence of increased blood flow to the lungs is well known. Therefore, in order to make a correct diagnosis in infants, heart catheterization and angiocardiography have to be applied in most cases of malformation of the heart. Till now, the examinations have been concentrated mainly on older children, i.e. from about 3-4 years of age, and adults, mainly because there are greater technical difficulties in using these complicated methods in infants than later in life. Later experiences, 
however, have shown that these methods can well be applied on infants (Ziegler, 1954) and it is, therefore, now an important pædiatric problem to get a clear picture of the value and possibilities of these methods of examination in this age group.

\section{TECHNIQUE OF EXAMINATION}

Anasthesia. Our examinations have been performed under full or partial anæsthesia. In most cases narcodorm (the sodium salt of $\beta$-bromallyl-isopropyl-N-methyl-barbituracid) is used rectally, whereby the suitable dose in the end seems to be about $2 \cdot 5-3 \mathrm{cg}$. per $\mathrm{kg}$. body weight from a 5 per cent solution, given in an equal volume of water. If required during the examination small intravenous injections can be added through the inserted catheter. In addition, local anæsthesia is used.

Narcodorm has, like all barbiturates, a distinct and unpleasant depressing effect on the respiration that in three of our cases has caused a temporary interruption of the respiration, so that the patients for a short time have been given artificial respiration without this stopping the planned course of the examination. All three were given $3 \cdot 5-5 \mathrm{cg}$. of narcodorm per $\mathrm{kg}$. body weight: after having reduced this dose to $2 \cdot 5-3 \mathrm{cg}$. per $\mathrm{kg}$. we saw no symptoms of cessation of respiration and the anæsthesia seemed to be adequate. In 14 of 33 patients examined who were given a maximum of $3 \mathrm{cg}$. narcodorm per $\mathrm{kg}$., there had to be added $0 \cdot 3-0.7 \mathrm{cg}$. per $\mathrm{kg}$. body weight intravenously through the catheter just before angiocardiography. It should be possible to perform intubation immediately, if necessary, but we have never used it. The infants come to the examination on an empty stomach (no food for the last four hours) and a water enema is given three hours before the rectal anæsthesia.

In infants and small children catheterization is best done through the saphenous vein and the inferior vena cava rather than through an antecubital vein (Ziegler, 1954). We have administered no drugs such as pronestyl or quinidine prophylactically. The catheter is inserted into the vena saphena magna, although the femoral vein has been used in four cases, as the saphenous vein was too narrow. During the catheterization there was a permanent drip of a physiological saline solution without heparin through the catheter. Catheterization was tried in 51 infants, with success in 50 of them. In two cases the examination could not be performed because of the small size of the saphenous vein, but one of these has later been catheterized through vena saphena on the other side without difficulty.

It is advisable to have continuous electrocardiographic observations routinely during cardiac catheterization and angiocardiography. Extrasystoles and/or tachycardia (more than 225 beats a minute) are common and may force interruption of the examination (5 out of 50 cases). In 2 of these 5 cases paroxysmal ventricular tachycardia occurred and required withdrawal of the catheter to the vena cava and interruption of the examination and drug administration.

Angiocardiography. When the contrast medium has to be injected directly into the heart it is of vital importance that the catheters used are thin-walled enough. We have used the Lehmann catheter No. 5F, 6F, or 7F (United States Catheter Corporation, Glenn Falls, N.Y.; catheter No. $5 \mathrm{~F}$ used 13 times, No. 6F 35 times, and No. 7F twice). The amount of contrast injected has been 1.1-1.9 ml. per $\mathrm{kg}$. body weight-in half of the cases $1.5-1.6 \mathrm{ml}$. per $\mathrm{kg}$. body weight. Generally we have used Rheopak Astra, 70 per cent.

To obtain the maximum of information from this form of angiocardiography, the injection should be made rapidly and finished before the contrast material reaches the left side of the heart. This passage of the contrast medium requires about 3-4 heart systoles. As the heart rate in infants during these examinations range between 180 and 220 beats a minute, that is about 3 beats a second, it means that the contrast medium should be injected within a second. This is only possible when using special syringes, operated either by hand via a lever or better, as has been done in these examinations, with the help of a syringe operated by compressed air (Gidlund). The pressure used for injection has been $5-6 \mathrm{~kg}$. $/ \mathrm{cm} .^{2}$ The syringes in question are connected with the rœntgen apparatus in such a way that the exposures are automatically released when the injection is made. 
It is also necessary to take the X-ray pictures with very short intervals and preferably in two projections. We have used a cassette changer, manufactured by Georg Schönander in Stockholm, that has a rate of 6 pictures a second during 5 seconds in two positions at right angles to each other (a total of 60 pictures in 5 seconds).

Examples are given in Figs. 1-6.
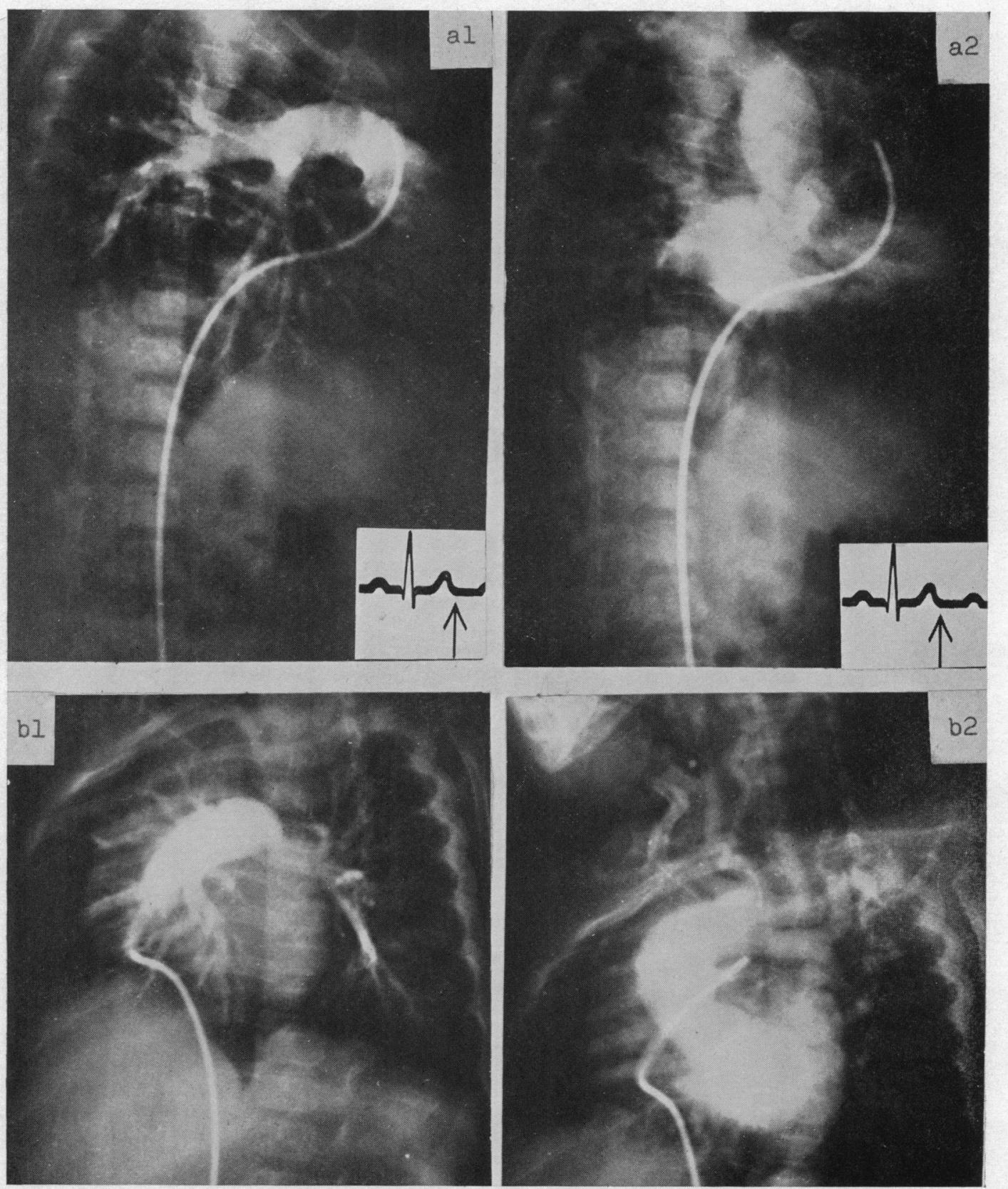

FIG. 1.-Coarctation of the aorta. Anterior oblique projections, right $\left(a_{1} a_{2}\right)$, left $\left(b_{1} b_{2}\right)$. Injection through a catheter into the pulmonary artery. $\left(a_{1} b_{1}\right)$ The pulmonary artery is intensely opacified and shows a normal pattern. The pulmonic valves are clearly seen $\left(a_{1}\right)$. $\quad\left(a_{2} b_{2}\right)$ The pulmonary circulation is completed 2.0 sec. later. Satisfactory opacification of the left side of the heart is seen. The ascending aorta is dilated. The length and degree of the narrowing of the coarcted area is clearly shown. 

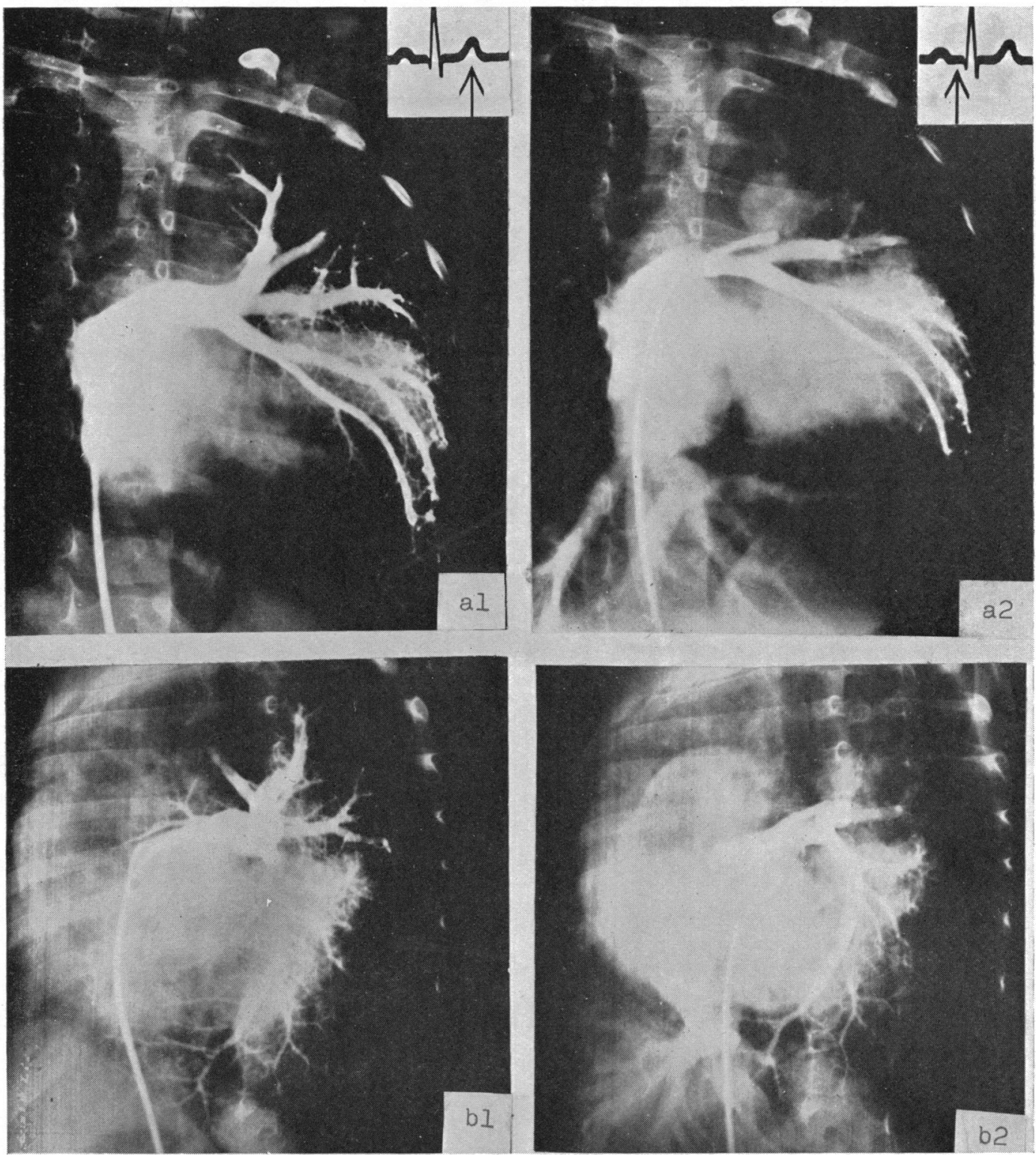

FIG. 2.-Atrial septal defect with a left-to-right shunt. Anterior oblique projections, right $\left(a_{1}-a_{2}\right)$, left $\left(b_{1}-b_{2}\right)$. Injection into the left atrium through a catheter. $\left(a_{1} b_{1}\right)$ The left atrium in diastole is well opacified. Some contrast is already seen in the right atrium $\left(b_{1}\right)$. There is intense retrograde filling of the left superior pulmonary vein. $\left(a_{2} b_{2}\right)$ In the following atrial systole a large shunt into the right atrium is seen. The right atrium is opacified to the same extent as the left. Reflux occurs into the inferior vena cava. 

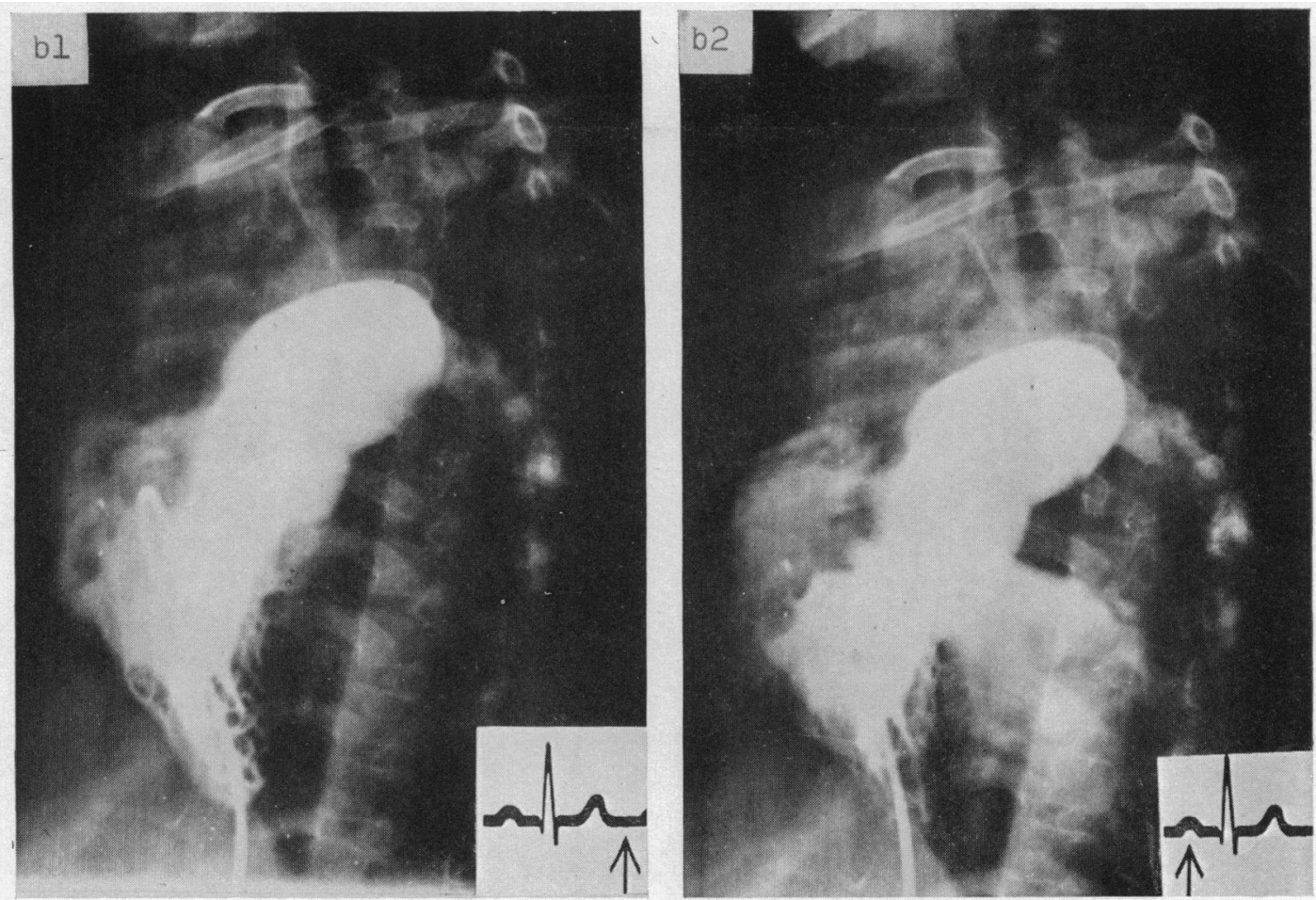

FIG. 3.-Ventricular septal defect. Left anterior oblique projection $\left(b_{1} b_{2}\right)$. Injection into the right ventricle through a catheter. $\left(b_{1}\right)$ The right ventricle and the pulmonary trunk are heavily contrast-filled. $\left(b_{2}\right)$ At the end of the same ventricular diastole a right-to-left interventricular shunt is seen. The size of the defect can be roughly estimated.
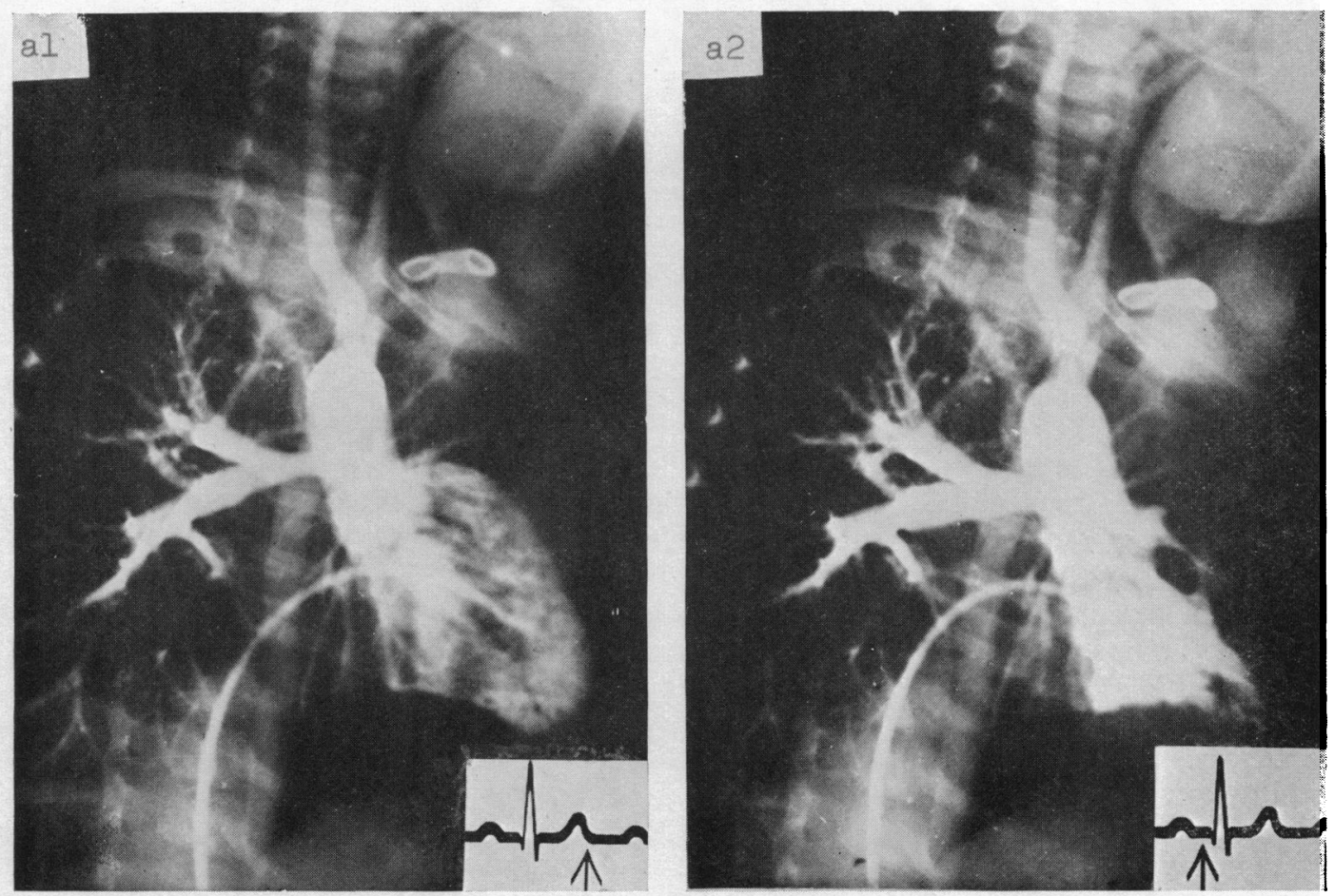

FIG. 4.-Tetralogy of Fallot. Right anterior oblique projections $\left(a_{1} a_{2}\right)$. Injection through a catheter into the right ventricle. $\left(a_{1}\right)$ The right ventricle is well filled with contrast. A broad aorta with an anteroposed origin is filled with contrast simultaneous with filling of the pulmonary artery. The infundibulum appears narrow and the main pulmonary artery is undersized. $\left(a_{2}\right)$ The infundibulum during ventricular systole is visualized as a thread-like lumen. There is oligamia of the peripheral lung fields. The pictures suggest that the infundibular stenosis is of functional significance only during ventricular systole. 


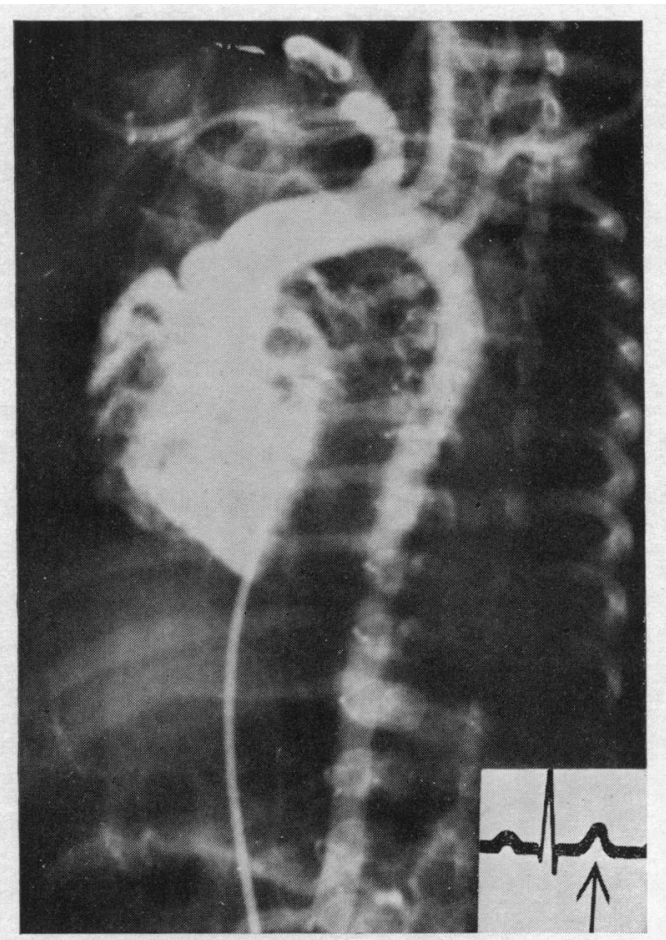

FIG. 5.-Transposition of the great vessels. Right anterior oblique projection. Injection was performed through a catheter directly into the right ventricle. This heart chamber is heavily opacified and shows at its border an irregular pattern, due to increased trabeculation in right ventricular hypertrophy. The aorta originates from the right ventricle and the arch is widely bowed. The coronary arteries are also visualized. No right-to-left interventricular shunt is seen. In the "aortic window" numerous fine vessels characteristic of bronchial collaterals can be seen.

\section{PATIENTS EXAMINED}

At Dronning Louises Børnehospital, Copenhagen, infants with congenital malformations of the heart have been examined by means of simultaneous heart catheterization and angiocardiography. with very rapid intracardial injection of contrast material. These investigations started in September 1954, and since then 50 infants have been examined: 8 of the children included in this material were more than 1 year of age (the oldest 1 year 11 months old), but have been included among the infants, as according to weight, they belong to the younger age group, the heaviest one weighing $9.7 \mathrm{~kg}$. The age and weight distribution is shown in Table I.

A preliminary diagnosis was made in each case based on a routine clinical examination and case history. The relation between the clinical diagnosis and the final diagnosis is seen in Table II. Septal defects were diagnosed clinically in 29 cases without specifying if localized to the atrial or to the ventricular septum as it is seldom possible to differentiate these in this very young age group. The diagnosis was correct in 26 of the cases but, as shown in Table II, incomplete. In 3 cases the left-to-right shunt was due only to a persistent ductus arteriosus. All cases considered, there have been 41 instances where the combination of clinical symptoms allowed a probable diagnosis to be 
TABLE I

Age AND Body Weight of Patients

\begin{tabular}{|c|c|c|c|c|c|c|c|}
\hline \multirow{2}{*}{\multicolumn{3}{|c|}{$\begin{array}{l}\text { Body weight } \\
\text { (kg.) }\end{array}$}} & \multicolumn{5}{|c|}{ Age (in months) } \\
\hline & & & 3 or less & $4-6$ & $7-9$ & $10-12$ & $12-24$ \\
\hline$<3.0$ & . & .. & 1 & & & & \\
\hline $3 \cdot 1-5 \cdot 0$ & . & . & 11 & 9 & & 1 & \\
\hline $5 \cdot 1-7 \cdot 0$ & . & . & & 8 & 2 & 4 & 1 \\
\hline $7 \cdot 1-9 \cdot 7$ & . & . & & & 4 & 2 & 7 \\
\hline & otal & .. & 12 & 17 & 6 & 7 & 8 \\
\hline
\end{tabular}

TABLE II

Comparison of Clinical Diagnosis and Final Diagnosis

\begin{tabular}{|c|c|c|c|c|c|}
\hline Clinical diagnosis & No. & Final diagnosis & No. & $\begin{array}{l}\text { Confirmed } \\
\text { by opera- } \\
\text { tion only }\end{array}$ & $\begin{array}{l}\text { Confirmed } \\
\text { by } \\
\text { autopsy }\end{array}$ \\
\hline $\begin{array}{l}\text { Septal defects with left-to-right } \\
\text { shunts. }\end{array}$ & 29 & $\begin{array}{l}\text { ASD } \\
\text { VSD } \\
\text { PDA } \\
\text { ASD+VSD } \\
\text { ASD+PDA } \\
\text { ASD+VSD+PDA } \\
\text { VSD ("Eisenmenger") } \\
\text { VSD with aorta from rudim. } \\
\text { outlet chamber } \\
\text { VSD+PDA+stenosis of aortic } \\
\text { valve+" infantile" type of } \\
\text { coarctation of aorta } \\
\text { PDA+PS }\end{array}$ & $\begin{array}{r}3 \\
11 \\
2 \\
6 \\
1 \\
1 \\
1 \\
2 \\
\end{array}$ & $\begin{array}{l}1 \\
3 \\
2\end{array}$ & $\begin{array}{l}4 \\
2 \\
1\end{array}$ \\
\hline $\begin{array}{lllll}\text { PDA } & \ldots & \ldots & \ldots & \ldots\end{array}$ & 1 & PDA & & 1 & \\
\hline Coarctation of aorta $\quad \ldots \quad \ldots$ & 1 & Coarctation of aorta & 1 & 1 & \\
\hline Tetralogy of Fallot $\quad \ldots \quad \ldots$ & 6 & $\begin{array}{l}\text { Tetralogy of Fallot } \\
\text { PS +PFO+PDA } \\
\text { Complete transposition of the } \\
\text { great vessels }\end{array}$ & $\begin{array}{l}4 \\
1 \\
1\end{array}$ & & $\begin{array}{l}3 \\
1\end{array}$ \\
\hline $\mathrm{PS}+\mathrm{ASD}$ & 1 & Tetralogy of Fallot & 1 & & 1 \\
\hline $\begin{array}{l}\text { Complete transposition of the } \\
\text { great vessels } \\
\text { Tricuspid atresia with under- } \\
\text { developed right ventricle }\end{array}$ & $\begin{array}{l}1 \\
1\end{array}$ & $\begin{array}{l}\text { Complete transposition of the } \\
\text { great vessels } \\
\text { As clinical diagnosis }+ \text { pulmon- } \\
\text { ary artery from left ventricle }\end{array}$ & $\begin{array}{l}1 \\
1\end{array}$ & & 1 \\
\hline Fibroelastosis $\quad \ldots \quad \ldots$ & 1 & Fibroelastosis + ASD & 1 & & \\
\hline Acyanotic, type uncertain & 6 & $\begin{array}{l}\text { Isolated PS } \\
\text { VSD } \\
\text { ASD+VSD } \\
\text { Uncertain }\end{array}$ & $\begin{array}{l}\frac{1}{2} \\
2 \\
1\end{array}$ & & $\begin{array}{l}1 \\
1\end{array}$ \\
\hline Cyanotic, type uncertain & 3 & $\begin{array}{l}\text { Tetralogy of Fallot } \\
\text { do. +PDA }\end{array}$ & $\begin{array}{l}2 \\
1\end{array}$ & & 1 \\
\hline Total $\ldots \quad \ldots$ & 50 & & 50 & 8 & 18 \\
\hline
\end{tabular}

Abbreviations: ASD: Atrial septal defects. PFO: Persistent foramen ovale. VSD: Ventricular septal defect. PDA: Persistent ductus arteriosus. PS: Pulmonary stenosis. 

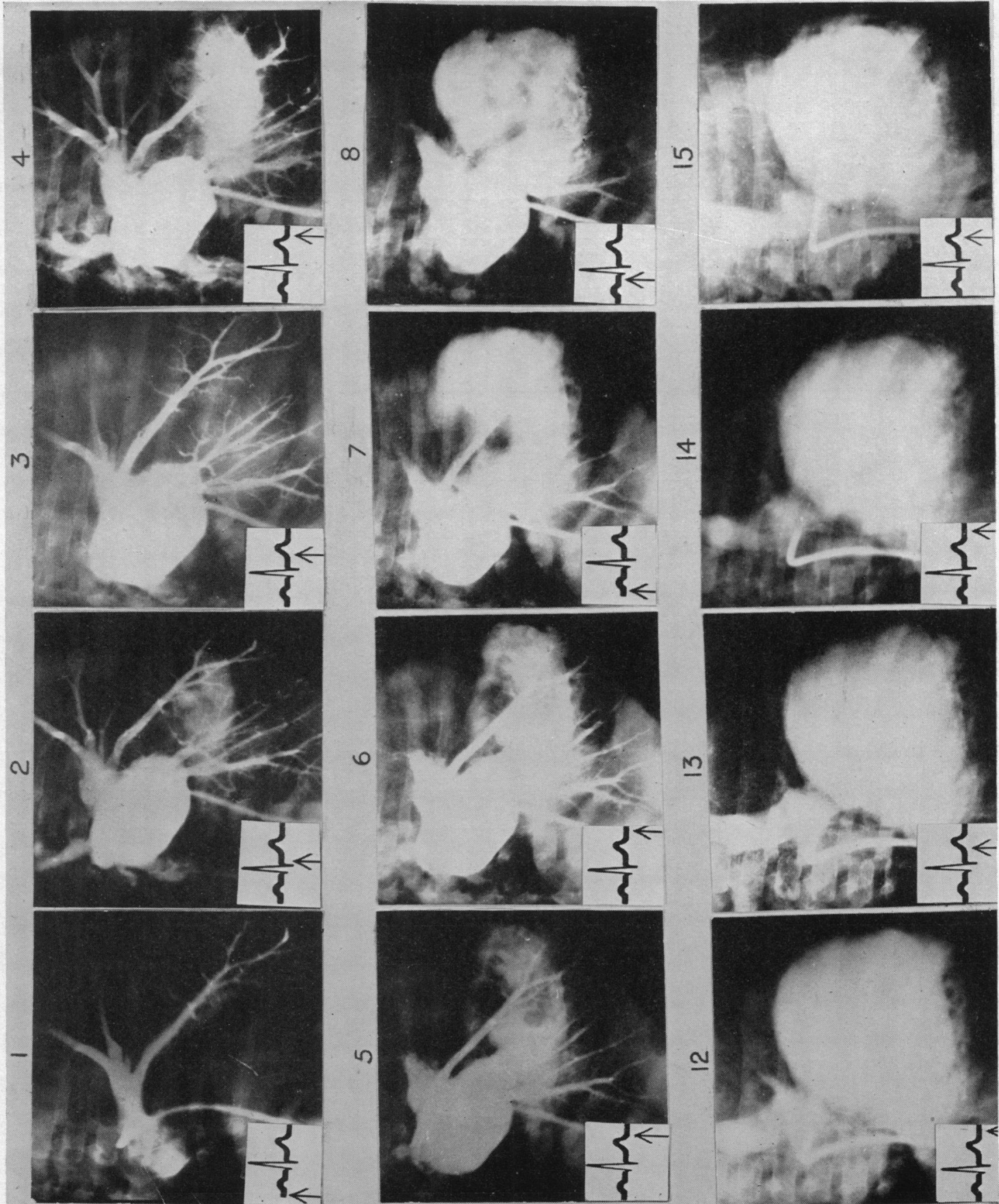

FIG. 6.-Fibroelastosis congenita (patent foramen ovale). Injection through a catheter into the left atrium. Right anterior oblique projection. The numbers above the pictures represent the real order of exposures. Rate of exposures 6 pictures per second. The series shows different successive stages of filling and emptying of the left atrium and ventricle. The slow filling and the exceedingly ineffective emptying of the left ventricle are strikingly illustrated. 
made. It is possible, therefore, in most cases to make a fairly correct diagnosis. Such a diagnosis is, however, usually not complete enough for surgical consideration, especially for the cases with leftto-right shunts. Complementary examinations are, as a rule, necessary to this end.

Risks. Two patients died the first day after the examination. In the first the examination itself may have aggravated an already pronounced insufficiency to such an extent that the infant could not be saved: the patient was exceptional in being under ether narcosis and was troubled by it, being coughing and restless. The other was a one-month-old boy who died suddenly the day after an intravenous angiocardiography which, in itself, had not affected him at all. According to the angiocardiograms, he probably suffered from a tricuspid stenosis but, unfortunately, the diagnosis was not verified as autopsy was not permitted. It is doubtful whether the angiocardiography could have caused the death. In this connection it should be mentioned that one patient died the day before the planned examination, and two on the morning of the day of the examination.

In one case the injection was unintentionally made into the coronary sinus, in one case into a pulmonary vein, and in one case through the heart muscle into the pericardium. Only the last patient was severely upset (shocked, and with ventricular tachycardia for 30 minutes).

\section{Discussion}

There are only a few publications available on the value of heart catheterization in infants. Ziegler (1954) has published the results of examinations of 188 children less than one year old and says that the method is simple and often gives quite satisfactory information. The method is, however, not discussed in detail. According to our findings it is hardly possible to transfer all the experiences of heart catheterization in adults to infants.

At heart catheterization three kinds of information are obtained, viz. (1) pressures from the different heart chambers and the great vessels; (2) oxygen saturation of the blood; and (3) abnormal communications that can be directly shown by the passage of the catheter. Concerning the pressure measurements in congenital heart diseases they are mainly of importance in the diagnosis of stenoses, but the presence of an increased pressure in the heart cavities in the absence of a stenosis is also of great interest. In 18 of 23 infants with ventricular septal defects with left-to-right shunts, there has been a considerably increased pressure in the right side of the heart (above $50 \mathrm{~mm} . \mathrm{Hg}$ ).

In our observations the pressure conditions in infants are rather unstable during catheterization: they often show considerable respiratory changes. The examinations are made under full or partial anæsthesia. There are thus no normal physiological conditions present. Future examinations may clarify the importance of these circumstances.

From determinations of the oxygen saturation of the blood at catheterization of normal subjects it has been found that the difference in oxygen saturation between the pulmonary artery and the right ventricle must be, at least, one volume per cent to permit the diagnosis of a left-to-right shunt. The same holds true about the difference between the right ventricle and the right atrium. Between the right atrium and the venæ cavæ the difference must be at least two volumes per cent. These values are obtained in older children and adults. No normal values are yet known for infants. According to our experience, these differences must be considerably higher in order to make a safe diagnosis of a left-to-right shunt, especially through an atrial septal defect.

Concerning the direct passage of a catheter through an abnormal communication between the two circulations, this has been a useful diagnostical method. In 15 of the 50 children examined the left atrium was catheterized, and only in 11 of these were there signs of an interatrial shunt. In 5 cases the aorta was catheterized through the ductus arteriosus.

We agree with Ziegler that heart catheterization in infancy is technically feasible and it is possible to get very valuable information concerning the anatomical diagnosis and the load on the circulation. This is, of course, of utmost value in deciding prognosis and therapy. 
Angiocardiography. If detailed information of the anatomical changes in the malformed heart is desired, the examination should be completed with angiocardiography.

Angiocardiography has been used to a great extent in the diagnosis of congenital heart diseases in infants (Castellanos et al., 1937; Lind and Wegelius, 1952). If the problem to be solved is physiological it presupposes that the contrast medium is injected intravenously because this way of introducing the contrast medium into the circulation causes least hæmodynamic disturbances. In a number of cases, however, this method does not give the detailed anatomical information the surgeon needs.

There are several advantages in injecting the contrast medium into the heart. In this way it is possible to get a higher concentration of the contrast material in the part of the heart that is of special interest to be visualized in each individual case. These requirements are best met with if the tip of the catheter lies in the chamber or vessel to be examined.

If the injection is made in the pulmonary artery the contrast reaches the pulmonary veins, the left atrium, and the left ventricle in high concentration. Anomalous pulmonary veins can in this way be visualized directly. A left-to-right shunt through an atrial or ventricular septal defect will be shown in the left anterior oblique projection, as the blood loaded with contrast medium is shunted from the left side of the heart into the right atrium or ventricle, empty of contrast material.

A satisfactory concentration of contrast medium in the aorta is also obtained by this method and this permits a study of the anatomy in cases of coarctation of the aorta.

Using intracardiac injection it seems possible sometimes to increase the pressure in the injected cavity and in this way artificially to reverse the shunt in cases of interventricular left-to-right shunts. By intravenous angiocardiography it is impossible to get exact information of the size and localization of the defect. By intracardiac injection of the contrast medium this may be possible in cases where a right-to-left shunt can be produced in ventricular diastole due to the rapid injection, but is not possible when the shunt is from the right atrium to the left, because in cases of atrial septal defects the left-to-right shunt is continuous through the whole heart cycle.

Finally, it must be stressed, as was originally done by Jönsson et al. (1949), that by this method it is possible to take full advantage of the results from the catheterization, considering both the pressures obtained in the various chambers, the oxygen content of blood withdrawn from them, and the rœntgenological aspects of the heart catheterization. Based on these results and the clinical examination, the injection of contrast medium can be carried out in a way that gives optimal information for the diagnosis and for an eventual operation.

\section{SUMMARY}

A combination of clinical diagnosis, heart catheterization, and rapid biplane angiocardiography with intracardiac injection of contrast medium has been used in the diagnosis of 50 cases of congenital heart diseases in infants. A satisfactory degree of certainty regarding both the pathological-anatomical changes, as well as the pressure and shunt conditions of the circulation has been obtained. After these investigations it has been possible to refer a number of infants for operation with a much more reliable diagnostic basis than previously.

\section{REFERENCES}

Castellanos, A., Pereiras, R., and Garcia, A. (1937). Arch. Soc. clin., 31, 523. Habana.

Jönsson, G. (1953). Modern Trends in Diagnostic Radiology. (Second Series), 114. Butterworth \& Co., London.

Lind, J. (1950). Acta Radiologica, Suppl., 82, Stockholm.

, and Wegelius, C. (1952). Advances in Pediatrics, 5, 154.

Ziegler, R. F. (1954). Pediatric Clinics of North America, 93. W. B. Saunders Co., Philadelphia and London. 